Sub. for publ.: Dec. 9, 2013; Rev.: Aug. 1, 2014; Re-rev.: Oct. 20, 2014;

Re-re-rev.: Apr. 13, 2015; Re-re-re-rev.: May 8, 2015

\title{
On the optimisation of a hybrid Tuned Mass Damper for impulse loading
}

\author{
J. Salvi ${ }^{1}$, E. Rizzi $^{1}$, E. Rustighi ${ }^{2}$, N.S. Ferguson ${ }^{2}$ \\ ${ }^{1}$ Dipartimento di Ingegneria, Università di Bergamo \\ viale G. Marconi 5, I-24044 Dalmine (BG), Italy \\ E-mail: jonathan.salvi@unibg.it; egidio.rizzi@unibg.it \\ ${ }^{2}$ Institute of Sound and Vibration Research, University of Southampton \\ Highfield, Southampton SO17 1BJ, UK \\ E-mail: er@isvr.soton.ac.uk; nsf@isvr.soton.ac.uk
}

\begin{abstract}
The present paper deals with the optimisation of a hybrid Tuned Mass Damper (TMD) in reducing the transient structural response due to impulse loading. In particular, a unit impulse excitation has been assumed, acting as base displacement, which is a situation that may occur in different real applications. The proposed hybrid Tuned Mass Damper is composed of a previously optimised passive TMD and an added optimised active controller. Such configuration has been conceived in view of reducing both the global and the peak response. Especially on the latter task, the introduction of the active controller brings in a significant contribution. Prior, a Bounded-Input-Bounded-Output (BIBO) stability analysis on the control gains is developed. Different control laws have been investigated, assuming as primary structures, first a single-degree-of-freedom (SDOF) benchmark system and then a multi-degree-of-freedom (MDOF) building, in order to point out the most appropriate control law for the given structural context. In particular, a new control law, based on a linear combination of acceleration and velocity, allowed for remarkable peak response reduction. The achieved dynamic response exhibits a time settling weakly oscillating response, an indication of a stable behaviour, and therefore represents a suitable option for the active controller, in view of various engineering applications.
\end{abstract}

Keywords: Hybrid Tuned Mass Damper (TMD); Optimum Tuning; Shock Loading; Unit Impulse Excitation; Feedback Closed-Loop Control. 


\section{Introduction}

The present work concerns the optimisation of hybrid Tuned Mass Damper devices for structural systems subjected to impulse loading. It is placed within a wider on-going research project [1-14].

Structural systems can be easily subjected to a wide range of harmful dynamical actions of a different nature, especially from the point of view of duration and intensity. Within this context, the reduction and control of the dynamic response due to impulse loading is doubtless an important research topic, mostly for its potential contribution in several engineering applications.

From their original introduction, which can be likely dated to more than one century ago with the patent of Frahm [15], Tuned Mass Dampers have always been one of the most studied control devices. In this sense, the main framework related to the passive TMD concerns its parametric optimisation, also called tuning, of its mechanical parameters, initially investigated by considering an undamped primary structure [16-18], and by assuming as external loading a harmonic excitation. Further studies deepened the knowledge of the optimum tuning of the passive TMD, by exploiting different response indices and extending the analyses to damped primary structures. In general, in such studies the structural system is assumed to be subjected to either harmonic [19-21] or white noise [22,23] excitations, acting either as a force on the primary structure or as base motion [3,24-26].

In recent years, semi-active, active and hybrid Tuned Mass Dampers have arisen in the field of structural control as complementary or alternative with respect to passive TMDs [27-29]. Such devices can change their governing parameters so as to extend their operating range and produce possible modifications of the structural behaviour. Moreover, the active controller is able to immediately supply a control force in order to respond to sudden changes within the dynamic context. Also for these studies, persistent signals of different characteristics have usually been considered, such as generic harmonic loading [30], wind excitation [31] or earthquake input $[32,33]$.

On the other hand, it appears that a comprehensive investigation for the case of shock loading, i.e. the optimisation of these control devices for the reduction of the transient structural response, is still lacking in the literature, even if some contributions have arisen for the passive TMD when applied to an undamped primary structure [34] or for the semi-active TMD [35]. However, the scenario of shock excitation covers an important case since it might provide a reference configuration to be identified for Tuned Mass Dampers when sudden excitations occur.

The present paper examines this case and deals with the study of the optimum tuning of a hybrid Tuned Mass Damper when the damped structural system is subjected to shock excitation. In particular, a structural system comprising of a damped SDOF primary structure and a hybrid TMD added on top, and subjected to a unit impulse acting as base displacement has been considered first.

The proposed hybrid TMD is composed of a passive TMD, previously optimised as outlined in [5], and a feedback closed-loop active controller, ruled by a control law that is a function of the system dynamic response. In this sense, besides preliminary considerations on the optimisation of the passive part of the control device, the main content of the present work focuses on the investigation into several possible control strategies and stability issues, aiming at outlining the best one for the considered structural context. The optimisation of the control gains is discussed in detail, with particular consideration to the efficiency in reducing the peak response, as compared to the required supplied control force.

Finally, the proposed tuning is extended to a case of a MDOF primary structure by taking a 10-storey shear-type frame building introduced in [38]. The related output confirmed the validity of the optimum control strategy for the proposed hybrid TMD. 


\section{Structural context and dynamic response}

\subsection{Structural system}

In the present study a structural system comprising a SDOF primary structure and an attached hybrid Tuned Mass Damper will be assumed as a benchmark model. This system is assumed to be initially at rest and subjected to a unit impulse excitation for $t=0$, which may be ideally defined by a Dirac delta function $\delta(t)$, characterised by the following properties [36,37]:

$$
\delta(t)=\left\{\begin{array}{cl}
\infty, & t=0 \\
0, & \text { elsewhere }
\end{array} \quad \int_{-\infty}^{+\infty} \delta(t) \mathrm{d} t=1\right.
$$

In particular, such impulsive excitation has been considered here acting as a base displacement $x_{g}(t)=X_{g} \delta(t)$, where $X_{g}$ denotes the constant amplitude of the excitation, assumed as unitary $X_{g}=1 \mathrm{~cm}$ in this study (Fig. 1). This case could likely represent a suitable model for sudden real dynamic loadings acting on mechanical systems or civil engineering structures.

The assumption of a SDOF primary structure is motivated by the aim of firstly outlining a general investigation into the proposed control laws and attached tuning method, whose validity is then finally assessed effectively (Section 4.5) for the case of a MDOF structure taken from [38].

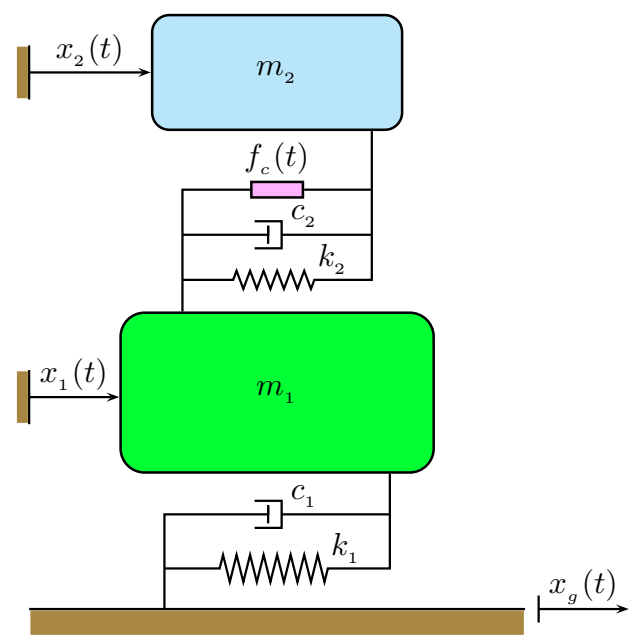

Figure 1: Structural parameters and absolute dynamic degrees of freedom of a 2DOF mechanical system, subjected to base displacement, comprising a SDOF primary structure (1), equipped with an added hybrid TMD (2), apt to supply a control force $f_{c}(t)$.

The mechanical parameters of such a structural system, which govern its dynamic behaviour, are given in the following. The primary structure is characterised by the mass $m_{1}$, the constant stiffness $k_{1}$ and the viscous damping coefficient $c_{1}$. The natural (angular) frequency $\omega_{1}$ and damping ratio $\zeta_{1}$ for the primary system are classically defined as follows:

$$
\omega_{1}=\sqrt{\frac{k_{1}}{m_{1}}}, \quad \zeta_{1}=\frac{c_{1}}{c_{1, c r}}=\frac{c_{1}}{2 \sqrt{k_{1} m_{1}}}=\frac{c_{1}}{2 \omega_{1} m_{1}}
$$

Similarly, the Tuned Mass Damper parameters are the mass $m_{2}$, the constant stiffness $k_{2}$ and the viscous damping coefficient $c_{2}$, while the relevant natural (angular) frequency $\omega_{2}$ and damping ratio $\zeta_{2}$ are consistently defined as follows:

$$
\omega_{2}=\sqrt{\frac{k_{2}}{m_{2}}}, \quad \zeta_{2}=\frac{c_{2}}{c_{2, c r}}=\frac{c_{2}}{2 \sqrt{k_{2} m_{2}}}=\frac{c_{2}}{2 \omega_{2} m_{2}}
$$


Two further parameters are introduced here, for the sake of comprehension of the following contents in this study, i.e. the mass ratio $\mu$ and the frequency ratio $f$ :

$$
\mu=\frac{m_{2}}{m_{1}}, \quad f=\frac{\omega_{2}}{\omega_{1}}
$$

The equations of motion of the considered 2DOF linear system can be stated in matrix form as follows:

$$
\mathbf{M} \ddot{\mathbf{x}}(t)+\mathbf{C} \dot{\mathbf{x}}(t)+\mathbf{K x}(t)=\mathbf{F}(t)+\mathbf{D} f_{c}(t)
$$

where:

$$
\mathbf{M}=\left[\begin{array}{cc}
m_{1} & 0 \\
0 & m_{2}
\end{array}\right], \quad \mathbf{C}=\left[\begin{array}{cc}
c_{1}+c_{2} & -c_{2} \\
-c_{2} & c_{2}
\end{array}\right], \quad \mathbf{K}=\left[\begin{array}{cc}
k_{1}+k_{2} & -k_{2} \\
-k_{2} & k_{2}
\end{array}\right]
$$

denote the structural matrices relevant to mass, viscous damping and elastic stiffness, respectively. The vectors:

$$
\mathbf{x}(t)=\left[\begin{array}{l}
x_{1}(t) \\
x_{2}(t)
\end{array}\right], \quad \dot{\mathbf{x}}(t)=\left[\begin{array}{l}
\dot{x}_{1}(t) \\
\dot{x}_{2}(t)
\end{array}\right], \quad \ddot{\mathbf{x}}(t)=\left[\begin{array}{l}
\ddot{x}_{1}(t) \\
\ddot{x}_{2}(t)
\end{array}\right]
$$

represent the dynamic response of the structural system, in terms of displacements, velocities and accelerations, respectively.

The vector of the dynamic excitation $\mathbf{F}(t)$, for impulse base displacement, takes the form:

$$
\mathbf{F}(t)=\left[\begin{array}{c}
c_{1} \dot{x}_{g}(t)+k_{1} x_{g}(t) \\
0
\end{array}\right]=\left[\begin{array}{c}
c_{1} X_{g} \dot{\delta}(t)+k_{1} X_{g} \delta(t) \\
0
\end{array}\right]
$$

The vector:

$$
\mathbf{D}=\left[\begin{array}{c}
-1 \\
1
\end{array}\right]
$$

defines the location vector for the control force $f_{c}(t)$, which is taken as a linear function of the displacement, velocity and acceleration terms in the dynamic response of the combined structural system, where the constant coefficient values of the combination are called gains [40].

\subsection{Adopted control laws}

The present investigation concerns mainly a theoretical target of different control laws, that are proposed and assessed for an innovative hybrid TMD, already optimised in its passive part. Optimum tuning of the TMD is derived for each considered control law. Different control strategies have been analysed, in order to locate the best choice for the considered structural context. Two final ones, both based on two gain variables are extensively analysed in the following.

Such control laws may be successively implemented through the placement of appropriate sensors, apt to measure selected response indexes. The other response quantities required by the actuator, in order to supply the required control force, could be obtained by means of either electrical differentiators or integrators [41-46]. However, the research contents in the following is focused on the theoretical framework, stability and optimisation of the control device, leaving specific practical features and experimental implementations to further studies.

A first control strategy, which for the sake of simplicity will be reported as "Control Law 1" (CL1), is merely based on the kinematic response of the primary structure, and starts from often adopted references in the literature [27,28]:

$$
f_{c}(t)=f_{c}^{C L}(t)=g_{a} \ddot{x}_{1}(t)+g_{v} \dot{x}_{1}(t)+g_{d} x_{1}(t)
$$


where $g_{a}, g_{v}$ and $g_{d}$ are three gain constants, related to acceleration, velocity and displacement of the primary structure, respectively. Preliminary optimisation tests developed in the present setting have pointed out that the acceleration gain $g_{a}$ appears to play a negligible role within the global amount of supplied control force $\left(g_{a}=0\right)$. Hence, the final representation of CL1 is considered in the following two-gain simplified version:

$$
f_{c}(t)=f_{c}^{C L 1}(t)=g_{v} \dot{x}_{1}(t)+g_{d} x_{1}(t)
$$

The second control strategy alternatively proposed in this study, which will be labelled "Control Law 2" (CL2), is based on the acceleration of the primary structure $\ddot{x}_{1}(t)$ and the relative velocity between the primary structure and the Tuned Mass Damper $\dot{x}_{1}(t)-\dot{x}_{2}(t)$ :

$$
f_{c}(t)=f_{c}^{C L 2}(t)=g_{a} \ddot{x}_{1}(t)+g_{v}\left(\dot{x}_{1}(t)-\dot{x}_{2}(t)\right)
$$

where $g_{a}, g_{v}$ are two gain constants related to the acceleration of the primary structure and the relative velocity, respectively. The introduction of this latter control law is motivated by the aim of controlling both the motion of the primary structure and the relative motion between structure and TMD, which under particular conditions could inadvertently amplify the motion of the primary structure instead of reducing it.

The extension of Eqs. (11)-(12) to the case of MDOF host structures is immediate and provides the following equations:

$$
\begin{aligned}
& f_{c}(t)=f_{c}^{C L 1}(t)=g_{v} \dot{x}_{n}(t)+g_{d} x_{n}(t) \\
& f_{c}(t)=f_{c}^{C L 2}(t)=g_{a} \ddot{x}_{n}(t)+g_{v}\left(\dot{x}_{n}(t)-\dot{x}_{n+1}(t)\right)
\end{aligned}
$$

for CL1 and CL2, respectively, where $n$ denotes the controlled $n$-th degree of freedom of the host structure, while $n+1$ is the degree of freedom of the attached TMD.

\subsection{Dynamic response}

The response of the structural system in the time domain to a true impulse loading (Dirac's delta function) has been obtained using a pair of Laplace transforms [37]. In this sense, a usual procedure for the solution of optimum control problems may concern the statement of the dynamic behaviour through a state-space model, which looks suitable for the adoption of control methodologies based on a set of first-order differential equations, such as for the Pole Placement method or the Linear Quadratic Regulator (LQR) method [40]. However, the Laplace transformation of the equation of motion has been adopted here as the most suitable approach, because it allows to model exactly the impulse excitation, shaped through a Dirac's delta distribution, that considers a respective Laplace-transformed form. Therefore, this process, while shall represent an alternative option in the field of control methodologies, it also ensures the whole truthfulness of the evaluation of the dynamic response to impulse loading. Moreover, this procedure results quite efficient from the computational point of view, since it envisages just the path transformation $\rightarrow$ multiplication $\rightarrow$ inverse transformation, which involves fast linear algebra steps, instead of heavier methods such as the numerical integration of the equations of motion (Newmark or Runge-Kutta integration methods) [37]. Nevertheless, a comparison of the proposed suitable procedure with a benchmark optimum control method, namely a LQR algorithm, has been outlined at this stage for first validation purposes, with related outcomes briefly discussed in Section 4.3.1.

Firstly, the equations of motion in the time domain of such a system subjected to a generic external excitation $f_{i}(t)$ are transformed in the Laplace variable $s$, with homogeneous conditions, consistent with the hypothesis of the system being initially at rest:

$$
\left[\begin{array}{ll}
Z_{11}(s) & Z_{12}(s) \\
Z_{21}(s) & Z_{22}(s)
\end{array}\right]\left[\begin{array}{l}
X_{1}(s) \\
X_{2}(s)
\end{array}\right]=\left[\begin{array}{l}
F_{1}(s) \\
F_{2}(s)
\end{array}\right]
$$


or, in compact form:

$$
\mathbf{Z}(s) \mathbf{X}(s)=\mathbf{F}(s)
$$

where $\mathbf{Z}(s)$ is the impedance matrix, $\mathbf{X}(s)$ is the degrees of freedom vector and $\mathbf{F}(s)$ is the excitation vector.

By considering that the Laplace transform of the unit impulse takes the form [40]:

$$
\mathcal{L}[\delta(t)]=1, \quad \mathcal{L}[\dot{\delta}(t)]=s
$$

the force vector becomes:

$$
\left[\begin{array}{l}
F_{1}(s) \\
F_{2}(s)
\end{array}\right]=\left[\begin{array}{c}
s c_{1} X_{g}+k_{1} X_{g} \\
0
\end{array}\right]
$$

Then, the dynamic response of the structural system in terms of the displacement as a function of $s$ can be obtained by an algebraic manipulation of Eq. (15):

$$
\mathbf{X}(s)=\mathbf{Z}(s)^{-1} \mathbf{F}(s)=\mathbf{H}(s) \mathbf{F}(s)
$$

where $\mathbf{H}(s)=\mathbf{Z}(s)^{-1}$ is the receptance matrix of the system.

By firstly considering CL1, the Laplace transform of Eq. (11) for a system initially at rest takes the form:

$$
F_{c}^{C L 1}(s)=s g_{v} X_{1}(s)+g_{d} X_{1}(s)
$$

The substitution of Eq. (19) into the transformed of Eq. (5), by considering Eqs. (15)-(18), gives the following impedance and receptance matrices:

$$
\begin{aligned}
& \mathbf{Z}_{C L 1}(s)=\left[\begin{array}{cc}
s^{2} m_{1}+s\left(c_{1}+c_{2}+g_{v}\right)+k_{1}+k_{2}+g_{d} & -s c_{2}-k_{2} \\
-s\left(c_{2}+g_{v}\right)-k_{2}-g_{d} & s^{2} m_{2}+s c_{2}+k_{2}
\end{array}\right] \\
& \mathbf{H}_{C L 1}(s)= \\
& =\frac{1}{\operatorname{det}\left(\mathbf{Z}_{C L 1}(s)\right)}\left[\begin{array}{cc}
s^{2} m_{2}+s c_{2}+k_{2} & s\left(c_{2}+g_{v}\right)+k_{2}+g_{d} \\
s c_{2}+k_{2} & s^{2} m_{1}+s\left(c_{1}+c_{2}+g_{v}\right)+k_{1}+k_{2}+g_{d}
\end{array}\right]
\end{aligned}
$$

Similarly, the Laplace transform of CL2, described in Eq. (12), for a system initially at rest, is the following:

$$
F_{c}^{C L 2}(s)=s^{2} g_{a} X_{1}(s)+s g_{v}\left(X_{1}(s)-X_{2}(s)\right)
$$

As before, the substitution of Eq. (22) into the transformed of Eq. (5), by considering Eqs. (15)(18), gives the following impedance and receptance matrices:

$$
\begin{aligned}
& \mathbf{Z}_{C L 2}(s)=\left[\begin{array}{cc}
s^{2}\left(m_{1}+g_{a}\right)+s\left(c_{1}+c_{2}+g_{v}\right)+k_{1}+k_{2} & -s\left(c_{2}+g_{v}\right)-k_{2} \\
-s^{2} g_{a}-s\left(c_{2}+g_{v}\right)-k_{2} & s^{2} m_{2}+s\left(c_{2}+g_{v}\right)+k_{2}
\end{array}\right] \\
& \mathbf{H}_{C L 2}(s)= \\
& =\frac{1}{\operatorname{det}\left(\mathbf{Z}_{C L 2}(s)\right)}\left[\begin{array}{cc}
s^{2} m_{2}+s\left(c_{2}+g_{v}\right)+k_{2} & s^{2} g_{a}+s\left(c_{2}+g_{v}\right)+k_{2} \\
s\left(c_{2}+g_{v}\right)+k_{2} & s^{2}\left(m_{1}+g_{a}\right)+s\left(c_{1}+c_{2}+g_{v}\right)+k_{1}+k_{2}
\end{array}\right]
\end{aligned}
$$

Finally, the transfer function relevant to the $i$-th degree of freedom can suitably be expressed in the following simplified form, based on a partial fraction expansion [40]:

$$
X_{i}(s)=G_{p} \sum_{n=1}^{N} \frac{R_{n}}{s-p_{n}}
$$


where $G_{p}$ is a constant gain factor, and the time-invariant amplitude of the input signal $R_{n}$ is a $n$-th constant called residue and $p_{n}$ is the $n$-th root of the denominator $D(s)$, also called pole of the system. Hence, the total number of poles $N$ corresponds to the degree of the denominator $D(s)$, which is the fourth order here. Such an expression for the transfer function is quite useful in view of the inverse Laplace transform, which returns the response in the time domain:

$$
x_{i}(t)=G_{p} \sum_{n=1}^{N} R_{n} \mathrm{e}^{p_{n} t}
$$

Despite that it could be possible, in principle, to derive the analytical expressions for the residues, even for a relatively simple system as that assumed in this study, such analytical expressions take quite complex and lengthy forms. Hence, the residues will always be evaluated numerically. At the same time, the poles could be evaluated analytically if the degree of the denominator is lower than five (Abel-Ruffini theorem), as it is for the case of the transfer function involved in this study (fourth order denominator). However, such analytical expressions are not strictly necessary for the present study, thus the evaluation of the poles will be carried out numerically as well.

\section{Bounded-Input-Bounded-Output stability analysis}

In this section, a preliminary stability analysis has been developed, so as to firmly establish the bounds (which will be further considered in the optimisation process) on the values that the control gains of the different feedback control laws may assume in order to ensure a priori a limited magnitude of the dynamic response in time for a given bounded input signal.

This principle is represented by the so-called Bounded-Input-Bounded-Output (BIBO) stability, which requires, as necessary and sufficient condition required for the stability of the motion of the system, negative real parts of the closed-loop poles [41], which for the structural system considered are the roots of the following characteristic equation:

$$
D(s)=0
$$

where $D(s)$ is the denominator of the transfer function, obtained as determinant of the impedance matrix $\mathbf{Z}(s)$. Such condition, in practical terms, ensures the decay of the amplitude of the vibrational modes of the structural systems.

In this sense, for Control Law 1, Eqs. (11), (19), the related characteristic equation becomes:

$$
\begin{aligned}
D_{C L 1}(s) & =\operatorname{det}\left(\mathbf{Z}_{C L 1}(s)\right)= \\
& =s^{4} m_{1} m_{2}+s^{3}\left(c_{1} m_{2}+c_{2} m_{1}+c_{2} m_{2}+g_{v} m_{2}\right)+ \\
& +s^{2}\left(c_{1} c_{2}+k_{2} m_{1}+k_{1} m_{2}+k_{2} m_{2}+g_{d} m_{2}\right)+s\left(c_{1} k_{2}+c_{2} k_{1}\right)+k_{1} k_{2}=0
\end{aligned}
$$

Similarly, for Control Law 2, Eqs. (12), (22), the following characteristic equation is obtained:

$$
\begin{aligned}
D_{C L 2}(s) & =\operatorname{det}\left(\mathbf{Z}_{C L 2}(s)\right)= \\
& =s^{4}\left(m_{1} m_{2}+g_{a} m_{2}\right)+s^{3}\left(c_{1} m_{2}+c_{2} m_{1}+c_{2} m_{2}+g_{v}\left(m_{1}+m_{2}\right)\right)+ \\
& +s^{2}\left(c_{1} c_{2}+k_{2} m_{1}+k_{1} m_{2}+k_{2} m_{2}+g_{v} c_{1}\right)+s\left(c_{1} k_{2}+c_{2} k_{1}+g_{v} k_{1}\right)+k_{1} k_{2}=0
\end{aligned}
$$

In order to satisfy the stability criterion described above, for each control strategy and set of given values of the gains, the sign of the less negative (or more positive) real part of the closed-loop poles of the system has been investigated, in order to establish a sort of stability threshold for the system. 
In this study, the fixed structural parameters of the primary system assumed in the numerical simulations are taken as:

$$
m_{1}=100 \mathrm{~kg}, \quad k_{1}=10000 \mathrm{~N} / \mathrm{m}
$$

leading to:

$$
\omega_{1}=10 \mathrm{rad} / \mathrm{s}, \quad f_{1}=\frac{\omega_{1}}{2 \pi}=1.592 \mathrm{~Hz}, \quad T_{1}=\frac{1}{f_{1}}=0.6283 \mathrm{~s}
$$

while the damping coefficient of the primary structure $c_{1}$ will take different values, so as to explore the influence of such parameter on the results. The structural parameters presented above have been chosen so as to easily allow for possible experimental validation on a real model, in order to check the preliminary results obtained here. In this sense, it is important to confirm that possible changes in the structural parameters should not affect the theoretical principles outlined within the present investigation.

The results of the analysis described above are represented in Fig. 2, where the stability regions are shown, for both considered control laws, as a function of the two control gains, for $\mu=0.02$ and $\zeta_{1}=[0.02,0.05]$. Such range of values can be considered as a suitable reference, and sufficient to outline important guidelines about the stability of the system.

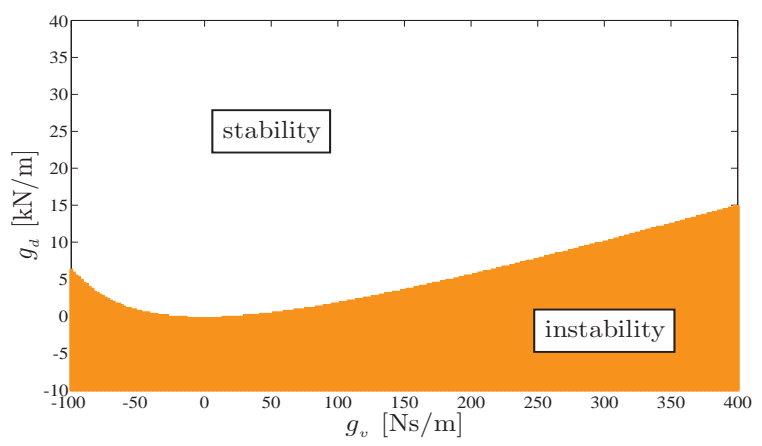

(a) $\mu=0.02, \zeta_{1}=0.02$.

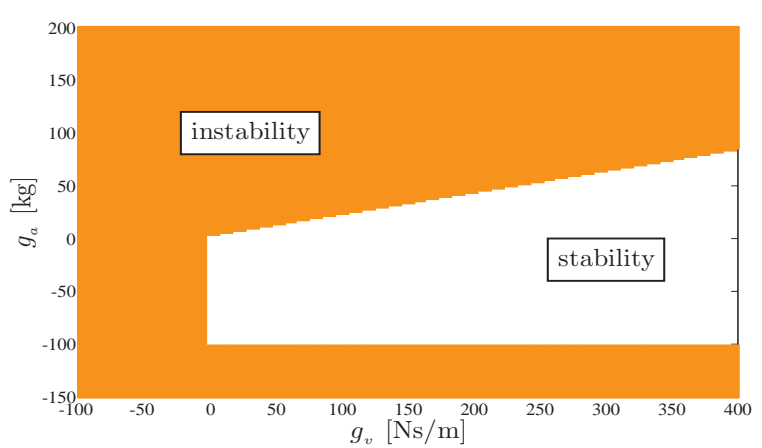

(c) $\mu=0.02, \zeta_{1}=0.02$.

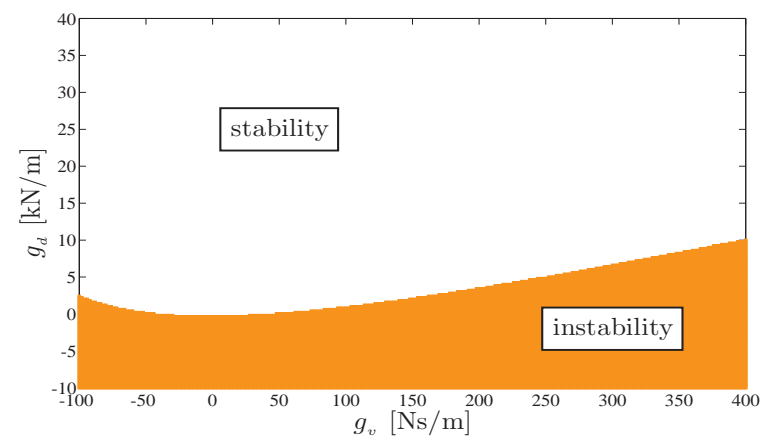

(b) $\mu=0.02, \zeta_{1}=0.05$.

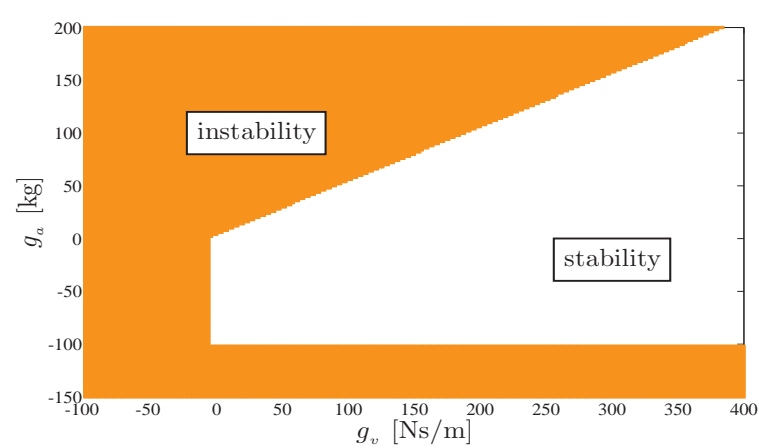

(d) $\mu=0.02, \zeta_{1}=0.05$.

Figure 2: BIBO stability region for (a),(b) Control Law 1 and (c),(d) Control Law 2, for mass ratio $\mu=0.02$ and different values of primary structure damping ratio $\zeta_{1}$.

The outcomes relevant to CL1 will be discussed first. From Figs. 2a-2b it can be noted that, as general consideration, the stability region is outlined by positive values of $g_{d}$, while an increase in the value of $g_{v}$ tends to narrow this area. It appears that the minimum values of the control gains assuring the stability of the system are of the same order of magnitude of 
the primary structure parameters $c_{1}, k_{1}$ (for $g_{v}, g_{d}$, respectively). Moreover, a brief comparison between Fig. 2a and Fig. $2 \mathrm{~b}$ points out that the higher the primary structure damping ratio, the wider the stability region.

The results related to CL2, reported in Figs. 2c-2d, exhibit a sharper contour of the stability region. This fact means basically that for a stable motion it is necessary to assume a positive value of $g_{v}$, while the acceptable range of values of acceleration gain $g_{a}$ is limited to negative values for $g_{v}=0$ and becomes larger, extended to positive values of $g_{a}$ at increasing $g_{v}$. Also, for CL2 a sort of stability threshold corresponding to $g_{a}=-m_{1}$ can be established, which apparently represents a constant outcome, independent of the assumed control law. A possible physical interpretation of such a result can be the following: for $g_{a}<-m_{1}$ one obtains a sort of second virtual primary system, which moves in the opposite direction with respect to the main one. Such mass magnitudes, the real and the virtual one, and most of all their interaction, may easily create dynamic instability within the global system. However, these conditions are likely far from real applications, since an inertial force of the same magnitude of that of the primary structure is not feasible, especially in the case of buildings and, in general, for large systems. As found previously for CL1, by observing Figs. 2c-2d also for CL2 the amplitude of the stability region increases at increasing $\zeta_{1}$.

\section{Optimum feedback control for the hybrid TMD}

\subsection{Optimisation of the passive TMD}

As briefly stated, the control device investigated in this study is composed of a passive TMD and an active controller. In particular, a first tuning of the passive TMD is developed for impulse excitation through a looped optimisation process $[2-5,7,8]$. Then, the optimum control law for the active controller is outlined here, based on the dynamic behaviour of the passive system. In this sense, the parameters displayed in Eqs. (3)-(4), which rule the behaviour of the passive TMD, will be taken here as an outcome of a previous optimisation process, carried out on the passive TMD only [5]. This is based on the minimisation of the $\mathrm{H}_{2}$ norm of the primary structure displacement $x_{1}(t)$, by means of a numerical algorithm for nonlinear constrained optimisation based on the Interior Point method [47].

The further improvement expected from the introduction of the feedback closed-loop active controller, whose optimisation is the topic of the present study, is represented by its capability to contribute to the response mitigation just after the beginning of the excitation. Such behaviour allows to achieve further significant benefits with respect to the case of the previously optimised passive TMD.

\subsection{Analysis of the optimisation process for the hybrid TMD}

The stability analysis developed and discussed in Section 3 has provided important guidelines towards the optimisation process of the control gains, especially concerning the bounds on the range of values that such gains may assume. As a further step before the optimisation of the control gains, first a preliminary analysis of the objective function for both control laws will be presented, in order to better define the context and, most important, to check if the present optimisation problem is well posed. The optimisation of the control gains has been based on the evaluation of a selected norm of time response quantity, which is evaluated through a pair of Laplace transform, as stated in Section 2.3.

In this sense, the optimisation framework consistently follows from the procedure adopted for the evaluation of the dynamic response, based indeed on the Laplace transformation of the equations of motion, and allows to locate the optimum region of the assigned objective function. 
Hence, the presented procedure intrinsically ensures the best results in terms of pure abatement of mechanical response and of control process robustness, while other methods could result more appropriate in case of a multi-objective control optimisation. In this sense, different optimum control methods, including those usually adopted in the literature $[27,33]$, could be considered, such as the LQR algorithm [40], which operates on systems described by state-space models. This has been considered here just for comparison purposes (see discussion in Section 4.3.1).

The objective function considered for this study is the peak displacement of the primary structure $x_{1}(t)$ :

$$
\mathrm{J}(\mathbf{v})=\left\|x_{1}(t)\right\|_{\infty}
$$

where $\mathbf{v}$ is the vector of the control gains, which play the role of optimisation variables. The minimisation of the peak displacement is motivated by the fact that the passive Tuned Mass Damper has already been tuned by considering the $\mathrm{H}_{2}$ norm, namely the overall response of the primary structure, and it turned out actually unable to reduce the peak of response as well [5]. This is, in the end, one main motivation in the addition of the active controller to the existing system with optimum passive TMD.

A significant extract of the results of this investigation have been reported in Figs. 3-4, leading to the following considerations.

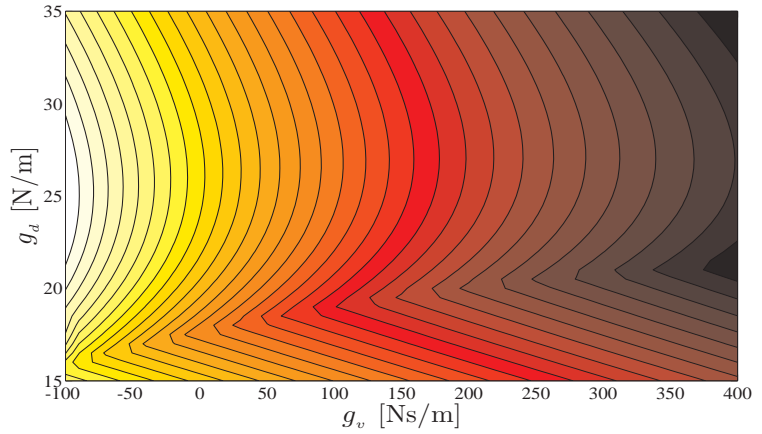

(a) $\mu=0.02, \zeta_{1}=0.02$

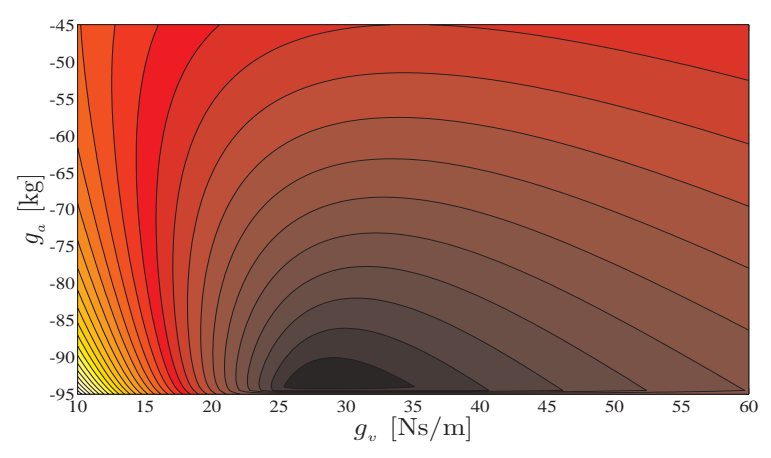

(c) $\mu=0.02, \zeta_{1}=0.02$

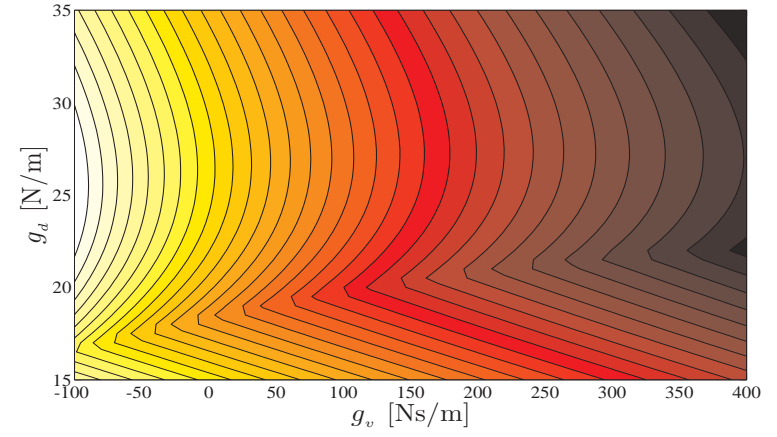

(b) $\mu=0.02, \zeta_{1}=0.05$

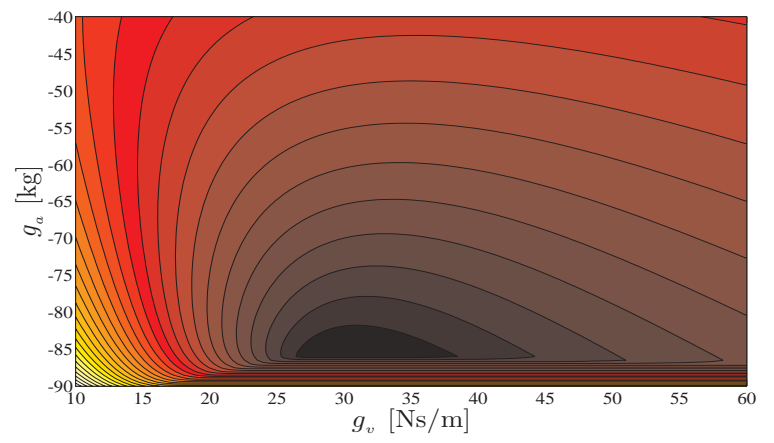

(d) $\mu=0.02, \zeta_{1}=0.05$

Figure 3: Objective function peak displacement of the primary structure for (a),(b) Control Law 1 and (c),(d) Control Law 2, as a function of the control gains, for $\mu=0.02$ and different values of primary structure damping ratio $\zeta_{1}$.

First, Control Law 1 is characterised by the damping gain $g_{v}$ and the stiffness gain $g_{d}$, which play here the role of optimisation variables:

$$
\mathbf{v}_{C L 1}=\left[g_{v} ; g_{d}\right]
$$


In this sense, Figs. 3a-3b represent the shape of the objective function for CL1, which basically takes smaller values for higher velocity gain $g_{v}$, whilst less sensitivity is ascertained for the acceleration gain $g_{a}$, since an actual minimum point is recovered for values almost twice the stiffness of the primary structure $k_{1}$, but such a minimum area tends to be enlarged at increasing $g_{v}$. From these features and previous stability analysis, the values of the bounds on the two control gains have been assumed as follows:

$$
0<g_{v}<50 \mathrm{Ns} / \mathrm{m}, \quad 10000 \mathrm{~N} / \mathrm{m}<g_{d}<25000 \mathrm{~N} / \mathrm{m}
$$

In particular, the upper bound on $g_{v}$ has been fixed since a clear minimum point corresponding to this control gain appears to be undefined, and also in order to limit the motion of the TMD, which may lead to large magnitude for higher values of the control gain. This fact could be physical explained by a motion of the TMD progressively joined with that of the primary structure, which for high values of $g_{v}$ may lead to system instability.

CL2 is based instead on the mass gain $g_{a}$ and the damping gain $g_{v}$, which play here the role of optimisation variables:

$$
\mathbf{v}_{C L 2}=\left[g_{a} ; g_{v}\right]
$$

The results in Figs. 3c-3d display an objective function characterised by a clear minimum area corresponding to a value of $g_{v}$ about one third of the primary structure damping coefficient $c_{1}$ and a value of $g_{a}$ slightly lower than the threshold value, obtained from the stability analysis, $g_{a}=-m_{1}$. In general, the presence of a well defined minimum area may lead already to consider this control law better than the previous one, at least from the point of view of the optimisation process, which should therefore result better posed.

Finally, the plots and previous stability analysis suggest the following lower and upper bounds for the two control gains:

$$
-95 \mathrm{~kg}<g_{a}<-75 \mathrm{~kg}, \quad 20 \mathrm{Ns} / \mathrm{m}<g_{v}<45 \mathrm{Ns} / \mathrm{m}
$$

The validity of the optimisation outcomes presented in the following sections, and the subsequent achieved dynamic response, will be evaluated also with respect to the results previously obtained for the passive TMD only [5], so as to point out the further benefit coming from the addition of the active controller.

\subsection{Optimum control gains and hybrid TMD performance}

The numerical results of the optimisation process on the control gains, for the considered case, have been summarised in Table 1, where the structural parameters, the optimum values of the control gains and the relevant percentage of response reduction are reported. These outcomes have been obtained by means of a Sequential Quadratic Programming (SQP) algorithm, and then validated with different other optimisation methods, such as: Genetic Algorithm, Simulated Annealing, Pattern Search Method [47]. Hence, the results presented here shall be characterised by significant robustness in terms of both concept and implementation.

Referring to Fig. 3, Fig. 4 further displays the trends of the objective function corresponding to the optimum value of $g_{v}$. The optimum control gains for CL1 exhibit a value corresponding to the upper value for $g_{v}$ and a value of $g_{a}$ increasing at increasing $\zeta_{1}$. In particular, the results on $g_{v}$ make necessary the assumption of an upper limit for this gain based on further features, besides the minimisation of the primary structure response. On the other hand, for CL2 a decreasing $g_{a}$ and an increasing $g_{v}$ at increasing $\zeta_{1}$ have been obtained, consistently with the preliminary analysis outlined before. Also, Fig. $4 \mathrm{~b}$ points out that for values even just lower 


\begin{tabular}{c|c|cc|cc}
\multicolumn{2}{c|}{\begin{tabular}{c} 
Control Law \\
\multicolumn{2}{c|}{ Primary structure damping ratio $\zeta_{1}$}
\end{tabular}} & 0.02 & 0.05 & 0.02 & 0.05 \\
\hline \hline Control & $g_{d}^{\text {opt }}[\mathrm{N} / \mathrm{m}](\mathrm{CL} 1), g_{a}^{\text {opt }}[\mathrm{kg}](\mathrm{CL} 2)$ & 18387.7 & 19128.7 & -94.2662 & -85.8264 \\
gains & $g_{v}^{\text {opt }}[\mathrm{Ns} / \mathrm{m}]$ & 50 & 50 & 28.2240 & 30.0005 \\
\hline & $\Delta\left\|x_{1}(t)\right\|_{\infty}$ & 39.66 & 38.98 & 28.09 & 24.25 \\
& $\Delta\left\|x_{1}(t)\right\|_{2}$ & 52.40 & 35.29 & 70.52 & 54.12 \\
Response & $\Delta\left\|x_{1}(t)\right\|_{\infty}-\Delta\left\|x_{1}(t)\right\|_{\infty}^{p}$ & 38.76 & 38.11 & 27.17 & 23.37 \\
reduction [\%] & $\Delta\left\|x_{1}(t)\right\|_{2}-\Delta\left\|x_{1}(t)\right\|_{2}^{p}$ & 15.55 & 16.29 & 33.67 & 35.12 \\
& $\Delta\left\|x_{2}(t)\right\|_{\infty}$ & 50.90 & 41.82 & 77.39 & 71.91 \\
& $\Delta\left\|x_{2}(t)\right\|_{2}$ & 63.35 & 58.65 & 89.05 & 85.81 \\
\hline Control & $\left\|f_{c}(t)\right\|_{\infty}$ & 1.07723 & 1.08793 & 34.3592 & 7.84583 \\
force $[\mathrm{kN}]$ & $\left\|f_{c}(t)\right\|_{2}$ & 30.7000 & 27.8247 & 53.1595 & 17.5010 \\
\hline
\end{tabular}

Table 1: Optimum control gains and response reduction $(\mu=0.02)$.

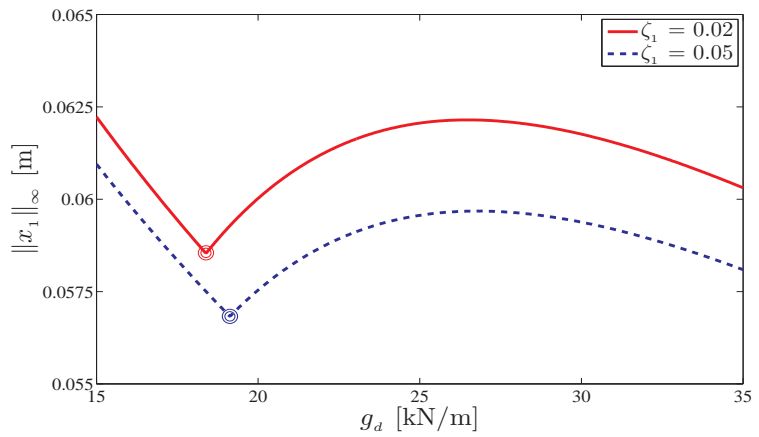

(a) CL1

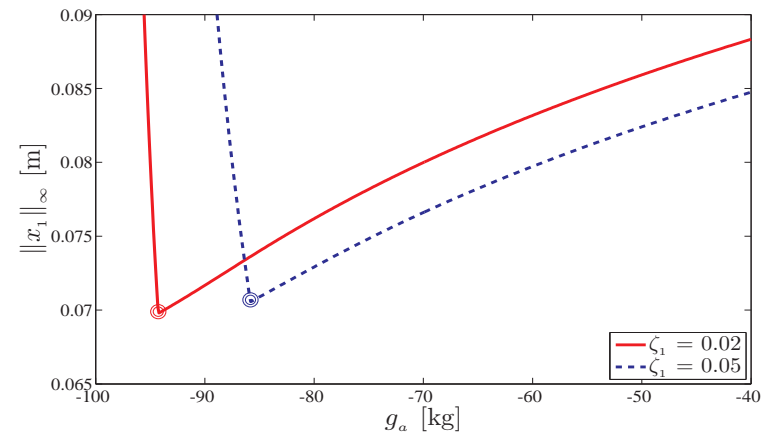

(b) CL2

Figure 4: Peak displacement of the primary structure at the optimum value of $g_{v}$, i.e. for (a) Control Law 1 at $g_{v}=50[\mathrm{Ns} / \mathrm{m}]$ and (b) Control Law 2 at $g_{v}=28.2240[\mathrm{Ns} / \mathrm{m}]$ with $\zeta_{1}=0.02$ and at $g_{v}=30.0005[\mathrm{Ns} / \mathrm{m}]$ with $\zeta_{1}=0.05$, as a function of the control gains, for $\mu=0.02$.

than that corresponding to the minimum, the peak displacement increases suddenly and hugely, likely due to the rising of the instability of the systems (Section 3).

Such values of the optimum gain could lead to the physical meaning that the active controller attempts to counteract the inertial force of the primary mass in the largest possible way, by trying to create a sort of "virtual" mass and, at the same time, it supplies a further quantity of damping between the passive TMD and the primary structure, so as to reduce the movement of the control device, which under particular conditions could amplify the response of the primary structure instead of reducing it.

The peak response of the primary structure, i.e. the $\mathrm{H}_{\infty}$ norm of $x_{1}(t)$, has been reduced significantly, i.e. of about $39 \%$ for CL1 and $24-28 \%$ for CL2, with a performance in general a bit lower for larger inherent damping, especially in the case of CL2. This is perhaps the most important outcome of the present analysis, since it strongly supports the introduction of the hybrid TMD for the purposes of the present control problem, i.e. the abatement also of the peak response. Remarkable results have been obtained also in terms of reduction of the $\mathrm{H}_{2}$ displacement, which in general decreases at increasing $\zeta_{1}$, especially for CL2. The difference 
in these indexes between the passive and the hybrid Tuned Mass Damper exhibits a general improvement in device performance [5]. Most of all, the reduction of the peak displacement appears to get greater improvement from the introduction of the active controller. On the other hand, the overall response, represented by the $\mathrm{H}_{2}$ norm, is reduced by a smaller amount. This is likely due to the previous beneficial effective optimisation already achieved with the passive TMD, based on this index. An important consequence and benefit due to the hybrid TMD is the large reduction of the TMD stroke, considered as either $\mathrm{H}_{\infty}$ or $\mathrm{H}_{2}$ norms. This fact is a very interesting and additional consequence of the optimisation process, even if the objective function has been established by neglecting the minimisation of the TMD response, and it could play an important role in view of practical applications.

The peak control force, which shall represent an important factor within the design of the active controller for the considered hybrid TMD (especially in terms of potential realisation and operational conditions), looks relatively small and almost constant for CL1, while it exhibits higher values for CL2, however largely decreasing at increasing inherent damping ratio $\zeta_{1}$, despite that for $\zeta_{1}=0.05$ the peaks of control force demanded by the two control laws still exhibit a remarkable difference. In this sense, the objective function has not concerned the limitation of the peak control force, if not through the bounds that are set on the gain variables. The present task concerns the highest gain that may be achieved in theoretical terms, specifically on the peak displacement response reduction of the primary structure. The overall control force $\left(\mathrm{H}_{2}\right)$ displays lower values for CL1 with respect to CL2 just at low damping, since the situation completely changes at increasing inherent damping. Indeed, for $\zeta_{1}=0.05$ the total amount of force required by CL2 is about one half of that computed for CL1. This means that a controller ruled by CL2 is able to take greater advantage from structural damping. Also, the higher peak force demanded by CL2 with respect to CL1 contributes to a more efficient abatement of the impulse response (see Figs. 5-6). This fact actually denotes finally a smarter behaviour of CL2, which aims at concentrating a large part of control force in cutting the peak response that could not be reduced by the passive TMD, while the control and reduction of the subsequent time response of the primary structure is managed by both the remaining control force and the contribution provided by the dynamic response of the optimised passive TMD. On the other hand, it could be noted that the control force related to CL1 is limited by the given upper bound on $g_{v}$, which looks as a consequence of the ill-posedness of the optimisation problem for CL1; indeed, for higher values on the upper bound for $g_{v}$, the quantity of supplied force for CL1 would increase significantly.

The dynamic responses of the different cases in the time domain, in terms of displacement of the primary structure and of the TMD, have been presented in Figs. 5-6. For CL1 (Fig. 5), the most noticeable fact is the almost equal shape of the dynamic response in the case of hybrid TMD. Indeed, it exhibits a sort of "double peak" at the very beginning of response, characterised by a constant amplitude, and the rest of response shows an oscillating behaviour, of higher period with respect to that of the passive system. Such results explain the numerical outcomes discussed above, since the amplitude of the peak response remains almost the same for the different cases, while the overall response decreases for increasing values of $\zeta_{1}$. As a consequence, the reduction in the peak response is a sort of constant result, while any further reduction of the overall response is smaller at higher $\zeta_{1}$.

The time histories for CL2 are presented in Fig. 6. The dynamic response of the hybrid TMD is characterised by a first peak in the response, followed by a rapid decrease in the oscillations, which end after about 2 seconds. In terms of the settling time this is a much more efficient behaviour with respect to the one obtained with the CL1, which leads to an oscillating primary structure for several seconds after the excitation. A further consideration regards the constant shape, from the point of view of the amplitude, of the minimised dynamic response. This was also found for the previous tests with CL1. 


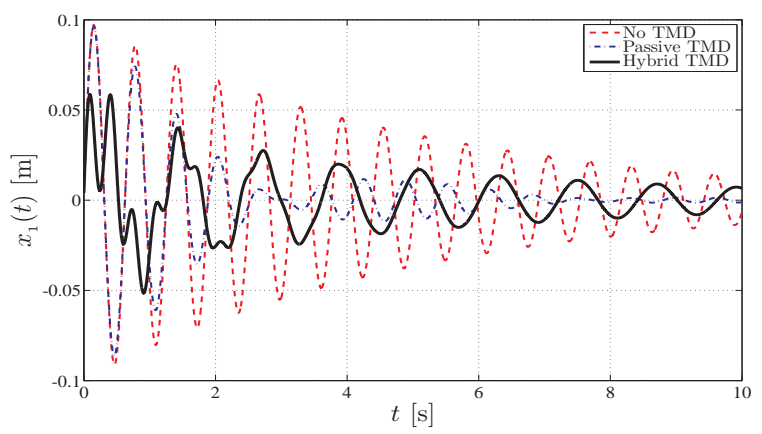

(a) $\mu=0.02, \zeta_{1}=0.02$.

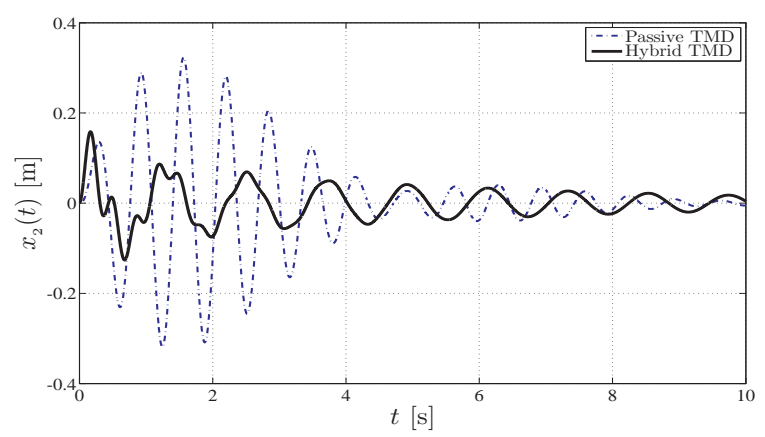

(c) $\mu=0.02, \zeta_{1}=0.02$.

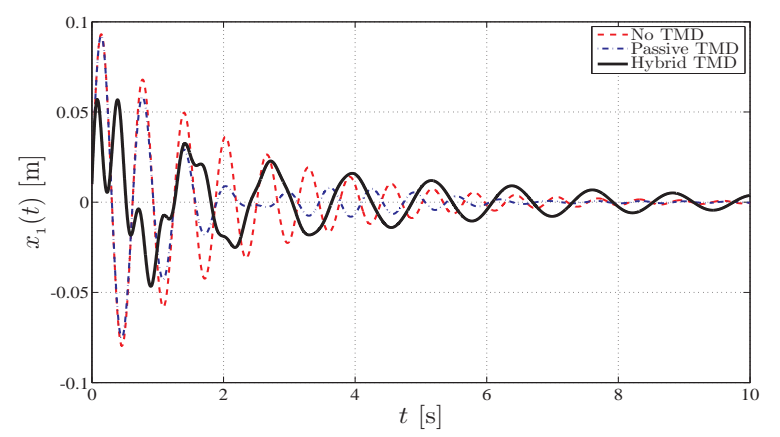

(b) $\mu=0.02, \zeta_{1}=0.05$.

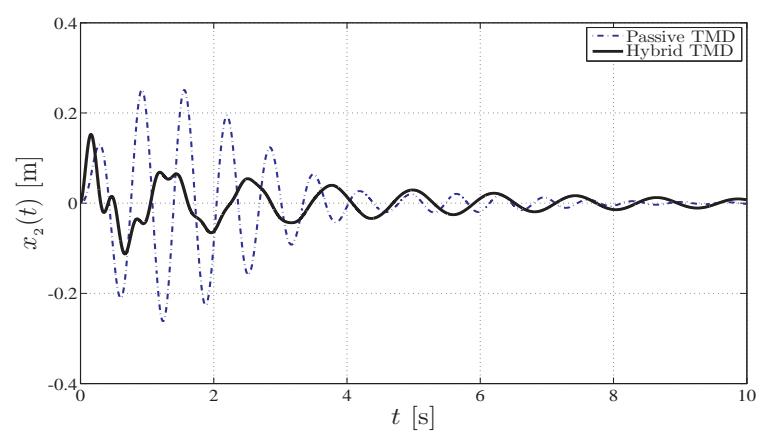

(d) $\mu=0.02, \zeta_{1}=0.05$.

Figure 5: Time history of (a),(b) the primary structure displacement $x_{1}(t)$ and $(c),(d)$ the TMD displacement $x_{2}(t)$, for Control Law 1, with and without TMD, for $\mu=0.02$ and different values of $\zeta_{1}\left(\zeta_{1}=0.02\right.$ in (a), (c), $\zeta_{1}=0.05$ in (b), (d)).

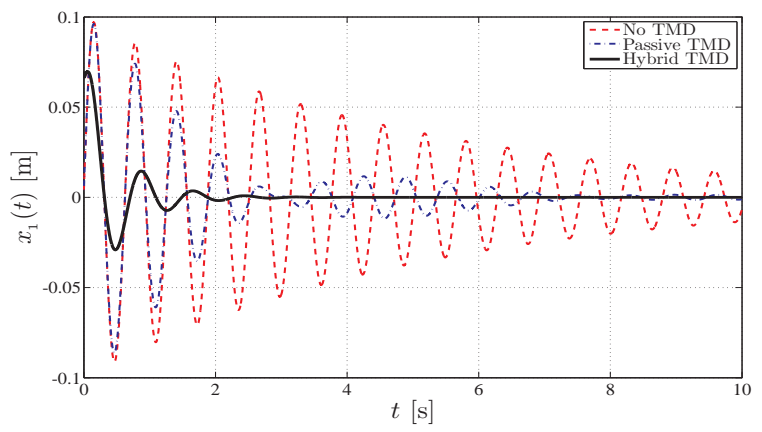

(a) $\mu=0.02, \zeta_{1}=0.02$.

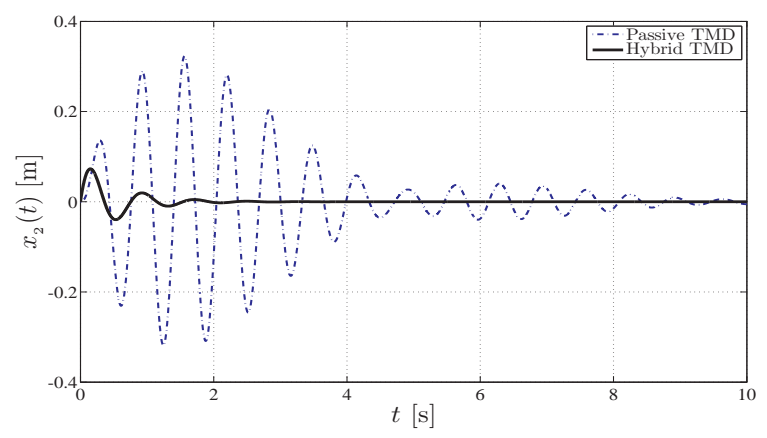

(c) $\mu=0.02, \zeta_{1}=0.02$.

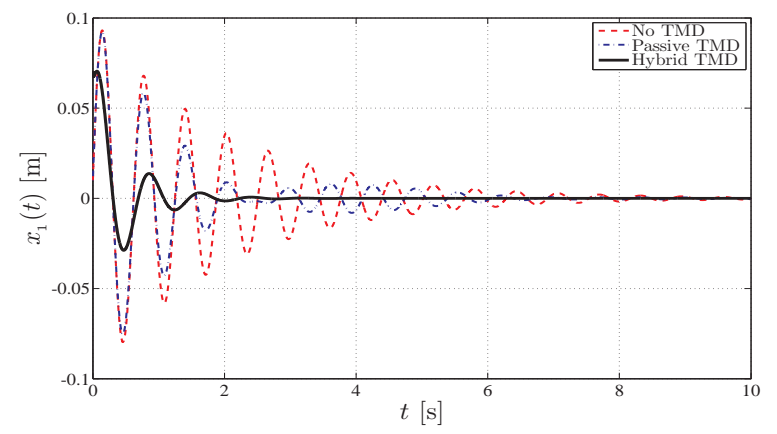

(b) $\mu=0.02, \zeta_{1}=0.05$.

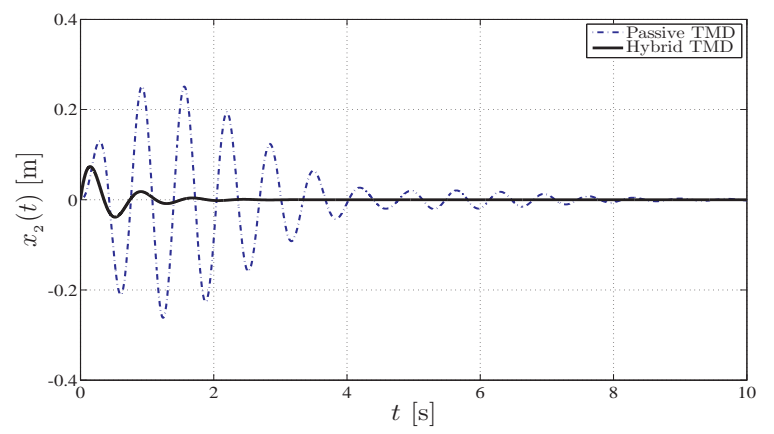

(d) $\mu=0.02, \zeta_{1}=0.05$.

Figure 6: Time history of (a),(b) the primary structure displacement $x_{1}(t)$ and $(c),(d)$ the TMD displacement $x_{2}(t)$, for Control Law 2, with and without TMD, for $\mu=0.02$ and different values of $\zeta_{1}\left(\zeta_{1}=0.02\right.$ in $(a),(c), \zeta_{1}=0.05$ in $\left.(b),(d)\right)$. 


\subsubsection{Comparison with a benchmark control methodology: LQR algorithm}

A comparison has been developed within the study of optimum control methodologies, by the adoption of a LQR method for the solution of the optimum control problem, with a setting of the weighting matrices aimed at the reduction of the impulse response, thus regardless of the limitation of the supplied control force, as done previously with the proposed method $[40,47]$.

Thereby, due to the state-space statement of the dynamic behaviour, the impulse excitation has been modelled as a versed-sine pulse $[4,36]$. The numerical trial pointed out a good effectiveness in reducing the tail of the time response, with oscillating behaviour of both the primary structure and the TMD (similar to the situation recovered for CL1). However, just a reduced effect on the abatement of the peak response has been recorded, especially if compared to the benefit achievable with the CL2-optimised hybrid TMD. This issue holds true concerning also the reduction of the TMD stroke.

Thus, such further results lead again to prefer the methodology proposed in this study, focused on a pure impulse excitation and therefore apt to provide the most effective control device for the given dynamic context and control problem.

\subsection{General closing considerations on the two assumed control laws}

The results obtained from the previous sections allow the following general remarks and considerations to be outlined, with the aim of establishing the best control strategy among the control laws analysed for the present structural context.

First of all, the issues of stability analysis in Section 3 and plots of the objective function in Fig. 3 show strong favour for newly-proposed CL2, which clearly displays different features typical of a well-posed optimisation problem.

The dynamic behaviour obtained by assuming the first control law (CL1), typically quoted in the literature, is characterised by two peaks of significant amplitude at the very beginning of the response time history, which could seriously jeopardise the resistance of the structure. Moreover, during the remaining dynamic response, the oscillating response exhibits non-negligible magnitudes.

On the other hand, the second control law (CL2) leads, in terms of dynamic response, to a unique initial peak, and then the remaining time history shows a response characterised by a rapid decay. Such a difference detected from the dynamic responses achieved by the two control laws points out the higher performance obtainable in case of the newly-proposed CL2, since the primary structure would be less stressed at all points in time.

Further, numerical results from Table 1, from the point the point of view of supplied control force, basically show what follows:

- Peak force $\left(\mathrm{H}_{\infty}\right.$ norm): almost constant and small for CL1 (due to the given bounds within the optimisation process), larger but strongly decreasing at increasing inherent damping for CL2;

- Overall force $\left(\mathrm{H}_{2}\right.$ norm): slightly decreasing at increasing damping for CL1, largely decreasing at increasing damping for CL2;

- Influence of inherent damping: the (unbounded) higher demand of control force required by CL2 occurs just for quite low damping values, i.e. $\zeta_{1}=0.02$ in this study. Indeed, for damping values close to those that may be typical of buildings and civil engineering structures (0.03-0.05), the amount of energy decreases dramatically, and the force required by CL2 becomes quite smaller than that demanded by CL1. For instance, with $\zeta_{1}=0.05$ the total supplied control force by CL2 is about one half of that required by CL1. 
Moreover, the higher peak force demanded by CL2 with respect to CL1 contributes to a more efficient abatement of the response (see Figs. 5-6). Hence, if for low damping there could be room for a contest between the two control strategies (and only in terms of the supplied control force), for $\zeta_{1}>0.02-0.03$ CL2 doubtless results in the best choice, among the presented control laws, from both qualitative and quantitative points of view. In this sense, a potential realisation of a controller ruled by CL2 should necessarily take into account the need of a significant and sudden power supply, so that to produce the expected performance in terms of maximum response abatement. Besides, in principle, it appears that CL2 turns out a control law apt to smartly manage both the action of the active controller and the contribution offered by the anti-resonant behaviour of the optimum passive TMD. Selected needs of bounding the amount of peak control force could be implemented within the present optimisation method, in view of specific requirements (e.g. costs) that may arise in real applications.

Finally, it should be considered that in the present context no attempts to optimise or to bound the amount of the required control force have been made. Control laws are just evaluated in terms of resulting displacement response of the primary structure. Further optimisation on this or other issues (e.g. control of the TMD stroke, etc.) could be possibly considered, within the same main implant, for additional tuning refinements based on selected needs depending also on requirements that may arise from practical applications.

\subsection{Real scenario application to a MDOF frame building}

The tuning developed on a SDOF+TMD structural system has been taken for exploring the optimisation of the controller under impulse loading. The assumption of a SDOF host structure plays a fundamental role in the present context, since it represents a basic model of a generic primary structure, useful to develop the tuning process and the control theory related to this study towards the optimisation of a hybrid TMD to be added on it. Moreover, such an approach is usually adopted in the literature, mostly to outline a new method of analysis or to state an innovative device [8-26]. Also, the SDOF system represents a valid model of structures fully characterised by a single bending vibration mode with maximum response on top, such as regular tall buildings. Notice that the optimisation of the active part of the TMD device is performed on the control gains by minimising interactively the response to impulse loading (i.e. not as post-tuning process).

The study is now extended, for CL2, by considering a realistic shear-type multi-storey (10 floors) frame building from the literature [38], with results in terms of response reduction that confirm the effectiveness of the present methodology, as proven on the original benchmark SDOF host structure. In practice, the looped reference optimisation procedure runs on a SDOFlike system but results in a TMD effectiveness that can be consistently assessed also for MDOF systems, as proven below.

In the considered building, the first mode of vibration turns out to be the dominant one within the dynamic response (with effective modal mass that is more than $80 \%$ of the total mass) and the modal frequencies are well separated. Hence, it is possible to make reference, just within the optimisation process, to a SDOF reference structure, as done earlier, with the parameters of the first vibration mode [24], which has been assumed as primary structure during the optimisation process. The so-obtained hybrid TMD is placed on top of the MDOF structure, where the maximum amplitude of dynamic response is expected.

Then, the dynamic analysis of the system, i.e. the evaluation of its dynamic response before and after the tuning of the device is developed by considering the actual MDOF host structure (10 degrees of freedom) + the TMD placed on top (11-th degree of freedom). The top floor displacement is therefore taken as the target degree of freedom to be controlled by a single hybrid TMD with a two-gain control force set on that. In this sense, scenarios of multiple TMDs 
have not been contemplated within this study. Hence, Eq. (13b) changes into the following:

$$
f_{c}(t)=f_{c}^{C L 2}(t)=g_{a} \ddot{x}_{10}(t)+g_{v}\left(\dot{x}_{10}(t)-\dot{x}_{11}(t)\right)
$$

First, the modal parameters related to the first mode of vibration, which represent a SDOFequivalent primary structure and therefore substitute the parameters in Eqs. (30)-(31), are the following [38]:

$$
m_{I}=1.10887 \times 10^{6} \mathrm{~kg}, \quad \omega_{I}=3.14391 \mathrm{rad} / \mathrm{s}, \quad \zeta_{I}=0.02
$$

The given value of the mass ratio is the same previously adopted, namely $\mu=0.02$, and based on the effective modal mass of the controlled mode ( $m_{I}$ in this case), as outlined in [39].

Second, the passive TMD is tuned by considering as objective function the $\mathrm{H}_{2}$ norm of the SDOF-equivalent primary structure displacement, as outlined in [5], by obtaining the optimum TMD frequency and damping.

Then, the two gains $g_{a}, g_{v}$ of the active controller are optimised by following the proposed method, explained in Sections 4.2-4.3. A preliminary stability analysis and a study on the objective function (recall that at this stage this is the $\mathrm{H}_{\infty}$ norm of the primary structure displacement) have been carried out, by providing the bounds assumed within the optimisation process. The following optimum control gains have been achieved by the presented looped optimisation process:

$$
g_{a}^{o p t}=-94254.0 \mathrm{~kg}, \quad g_{v}^{o p t}=83668.5 \mathrm{Ns} / \mathrm{m}
$$

which lead to a reduction of the primary structure displacement of about $25 \%$ and $58 \%$, for the peak and the overall indexes, respectively. The peak control force required by the system is characterised by the following maximum magnitude:

$$
\left\|f_{c}(t)\right\|_{\infty}=75.7598 \mathrm{kN}
$$

As expected, the results clearly denote a higher magnitude of required control force as attached to the increased mass of the present MDOF case. Also, a similar increase is recovered for the control force. However, the principle of benefit coming from the introduction of the hybrid TMD is confirmed, since both peak and overall time responses of the primary structure have been reduced. Also, a remarkable TMD response abatement has been recovered for this case, as obtained earlier.

The optimum hybrid TMD is finally placed on top of the MDOF primary structure, where the maximum displacement is expected to occur. The time history displacement of the MDOF primary structure top storey and of the passive and hybrid TMD are displayed in Figs. 7a-7b, respectively.

At this stage, the theoretical Dirac delta function of the previous impulse analysis has been suitably approximated in the numerical treatment by a versed-sine pulse [36], as described in [4]. Though this makes some difference in the resulting time history responses, the impulse excitation is reasonably represented as in a real loading scenario. Again, the behaviour of the structural system basically matches the previous case, with a dynamic response decaying quickly after the beginning of the event. Hence, the present example confirms all the positive features provided by CL2, which proofs itself as a valid solution for the problem of the impulse response control. 


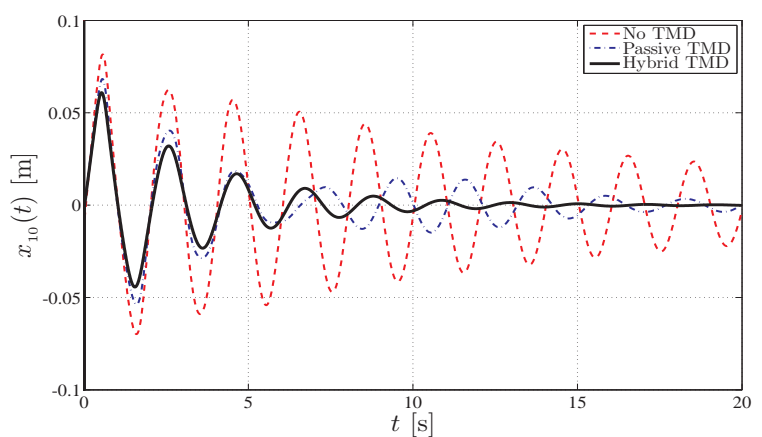

(a)

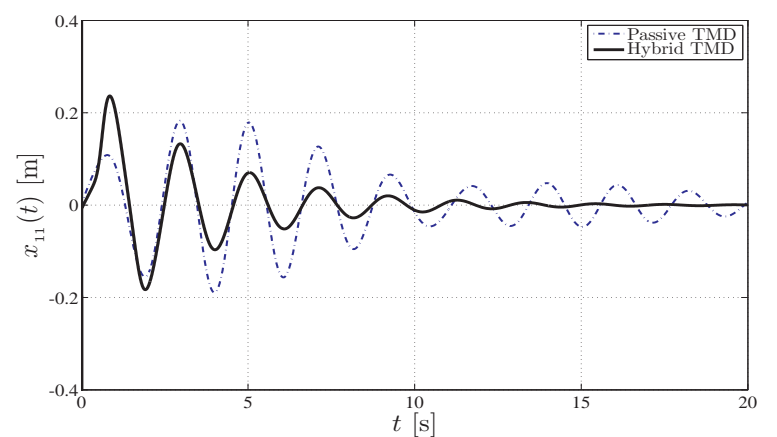

(b)

Figure 7: Time history of (a) the MDOF primary structure top storey displacement $x_{10}(t)$ and (b) the TMD displacement $x_{11}(t)$, for Control Law 2, with and without TMD ( $\left.\mu=0.02\right)$.

\section{Conclusions}

In the present work, the parametric optimisation of a hybrid Tuned Mass Damper device for systems subjected to unit impulse excitation has been investigated. In particular, the structural system is composed first of a reference SDOF primary structure, with a hybrid Tuned Mass Damper added on top of it. Later, results are assessed also with an application to a real MDOF structure.

The hybrid TMD is composed of a passive TMD, previously optimised in [5], so as to guarantee a basic performance in reducing the dynamic response of the structural system, and a feedback closed-loop active controller, which is assumed to be ruled by linear control laws, composed of quantities of the dynamic response and constant control gains. The introduction of the active controller was conceived in order to improve the performance of the solely passive TMD, especially in reducing the peak impulse response occurring at the very beginning of the time history.

Two control laws have been specifically presented and analysed, from both the points of view of the stability and of the optimisation of the control gains (two gains in both laws). The first one, adopted in the wake of literature proposals and named CL1, was composed of velocity and displacement of the primary structure, while the second one, newly-proposed control law, denoted CL2, was characterised by a different approach, ruled by a first control gain for the acceleration of the primary structure and a second gain related to the relative velocity between primary structure and TMD. The objective function for both control laws was based on the $\mathrm{H}_{\infty}$ norm of the peak displacement of the primary structure (which was not reduced by the passive TMD). An extensive BIBO stability analysis has been performed first on both control laws.

For the first control law (CL1), the optimum velocity gain exhibited values corresponding to the upper bounds, which have been assumed for the sake of motion stability, while the acceleration gain takes reliable values, within the assumed range. The improvement in the performance of the control device, especially in terms of reduction of the peak response, was considerable, and also the overall response of the primary structure and the stroke of the TMD have been further reduced significantly. However, this control strategy seems to exhibit also some relevant weaknesses, whose main ones are an unusual shape of the objective function, which could bring difficulties within the optimisation process, and a hard settlement in the time response, pointed out by an oscillatory behaviour that lasts along the whole response.

The second control law (CL2) was characterised by optimum values of the acceleration gain 
close to the lower stability bound, and quite negative anyway, and by a positive optimum damping gain. The effect of such a setting is a sort of virtual addition of a mass which moves in antiresonance with respect to the mass of the primary structure, while the relative movement between the primary structure and the TMD is reduced (or at least limited) by the further relative damping introduced. This control law leads to a significant abatement of the peak displacement, as obtained for the previous control law, even if the percentage reduction is a bit smaller than for CL1.

The amount of supplied control force required by CL2, a bit larger than that of CL1, for the peak supplied force and at small inherent damping, decreases largely at increasing inherent damping, and keeps respectful of the given bounds from the control gains. Also, the final exhibited time domain behaviour is much smoother and less oscillating. Indeed, CL2 tends to cut down the response since its very beginning, where a noticeable effort is required (whose amount of peak control force should be taken into consideration in view of practical realisations of a so-conceived hybrid device), and then it allows for a stronger decay of vibration amplitude. All such considerations lead to prefer CL2 with respect to CL1 for the present hybrid TMD.

Besides, the whole optimum tuning and control procedure proposed here, based on the classical statement of the dynamic behaviour through a set of second-order differential equations, and on the solution of both tuning and control problems by means of a non-linear optimisation algorithm, resulted in the best choice also in the light of alternative control methodologies, e.g. performed by means of a LQR algorithm. The latter provided a good validation in terms of reduction of the tail of the time response, but a much negligible effect in reducing the targeted peak response.

Finally, a further test has been carried out by assuming CL2 for the hybrid TMD added on top of a real 10-storey shear-type frame building, whose dynamic response is well represented by the first vibration mode, due to its intrinsic regularity. All the obtained results confirmed the features pointed out for the previous reference SDOF case, including the well posedness context of CL2 and, most important, the considerable reduction of the global structural response.

Among the salient achievements of the present study, main issues could be itemised as follows:

- A remarkable reduction of the peak response has been achieved for both two-gain control laws, which fact should, in principle, encourage the adoption of active controllers for the control of sudden excitations.

- A significant abatement of the TMD stroke has been obtained as a positive side effect, since such kinematic index has not been directly targeted within the looped optimisation process under impulse loading.

- In general, the newly-proposed CL2 provides better results and reliable behaviour with respect to the more traditional CL1, originated from the whole kinematic response of the primary structure.

- The present theoretical setting and achieved consistent results support further adoption and implementation in practical realisations.

\section{Acknowledgements}

The Authors would like to acknowledge public research funding from "Fondi di Ricerca d'Ateneo ex 60\%", at the University of Bergamo, Department of Engineering (Dalmine), a ministerial doctoral grant at the MITIMM Doctoral School of the same and research project funding " $F Y R E$ - Fostering Young REsearchers" supported by Fondazione Cariplo to the University of Bergamo, which allowed for exchange research at the Institute of Sound and Vibration Research (ISVR), University of Southampton. 


\section{References}

[1] E. Rizzi, D. Brescianini, M. Scotti, On the optimal tuning of Tuned Mass Dampers in structural systems, Proc. of ECCOMAS Thematic Conference - 2nd Int. Conf. on Computational Methods in Structural Dynamics and Earthquake Engineering (COMPDYN 2009), Rhodes, Greece, 22-24 June 2009, M. Papadrakakis, N.D. Lagaros, M. Fragiadakis (Eds.), 24 pages, 2009.

[2] J. Salvi, E. Rizzi, Minimax optimization of Tuned Mass Dampers under seismic excitation, Proc. of 8th Int. Conf. on Structural Dynamics (EURODYN 2011), Leuven, Belgium, 4-7 July 2011, G. De Roeck, G. Degrande, G. Lombaert, G. Müller (Eds.), Book of Abstracts, ISBN: 978-90-760-1931-4, p. 68; CD-ROM Proceedings, p. 1892-1899, 8 pages, 2011.

[3] J. Salvi, E. Rizzi, A numerical approach towards best tuning of Tuned Mass Dampers, Proc. of 25th Int. Conf. on Noise and Vibration Engineering (ISMA 2012), Leuven, Belgium, 17-19 September 2012, P. Sas, D. Moens, S. Jonckheere (Eds.), Book of Abstracts, ISBN: 978-90-738-0289-6, p. 141; CD-ROM Proceedings, p. 2419-2434, 16 pages, 2012.

[4] J. Salvi, E. Rizzi, E. Rustighi, N.S. Ferguson, Analysis and optimisation of Tuned Mass Dampers for impulsive excitation, Proc. of the 11th Int. Conf. on Recent Advances in Structural Dynamics (RASD 2013), Pisa, Italy, July 1-3, 2013, E. Rustighi (Ed.), Book of Abstracts, p. 64, CD-ROM Proceedings, ISBN: 9780854329649, Paper ID 1002, p. 1-15, 15 pages, 2013.

[5] J. Salvi, E. Rizzi, E. Rustighi, N.S. Ferguson, Best tuning of passive Tuned Mass Dampers for the mitigation of the impulse response, Preprint, 2013.

[6] J. Salvi, "Optimisation of Tuned Mass Damper Devices Towards Structural Vibration Reduction: Theoretical Settings and Numerical Analyses", Doctoral Thesis in Mechatronics, Informatics, Innovative Technologies and Mathematical Methods, Università di Bergamo, Dipartimento di Ingegneria (Dalmine), Italy, Advisor: E. Rizzi, 270 pages, 8th May 2014.

[7] J. Salvi, E. Rizzi, M. Gavazzeni, "Analysis on the optimum performance of Tuned Mass Damper devices in the context of earthquake engineering", Proc. of the 9th International Conference on Structural Dynamics (EURODYN 2014), Porto, Portugal, 30 June-2 July 2014, A. Cunha, E. Caetano, P. Ribeiro, G. Müller (Eds.), Book of Abstracts, ISBN: 978972-752-166-1, p. 85; CD-ROM Proceedings, ISSN: 2311-9020, ISBN: 978-972-752-165-4, p. 1729-1736, 8 pages, 2014.

[8] Salvi J, Rizzi E. "Optimum tuning of Tuned Mass Dampers for frame structures under earthquake excitation", Structural Control and Health Monitoring, doi: 10.1002/stc.1710, 22(4):707-715, 2015.

[9] E. Rustighi, M.J. Brennan, B. Mace, A shape memory alloy adaptive tuned vibration absorber: design and implementation, Smart Materials and Structures, 14(1):19-28, 2005.

[10] E. Rustighi, M.J. Brennan, B. Mace, Real-time control of a shape memory alloy adaptive tuned vibration absorber, Smart Materials and Structures, 14(6):1184-1195, 2005.

[11] C. Carmignani, P. Forte, E. Rustighi, Design of a novel magneto-rheological squeeze-film damper, Smart Materials and Structures, 15(1):164-170, 2006.

[12] B. Yan, M.J. Brennan, S.J. Elliott, N.S. Ferguson, Active vibration isolation of a system with a distributed parameter isolator using absolute velocity feedback control, Journal of Sound and Vibration, 329(10):1601-1604, 2010.

[13] S.-J. Jang, M.J. Brennan, E. Rustighi, H.-J. Jung, A simple method for choosing the parameters of a two degree-of-freedom tuned vibration absorber, Journal of Sound and Vibration, 331(8):4658-4667, 2012.

[14] M. Zilletti, S.J. Elliott, E. Rustighi, Optimisation of dynamic vibration absorbers to minimise kinetic energy and maximise internal power dissipation, Journal of Sound and Vibration, 331(18):4093-4100, 2012. 
[15] H. Frahm, Device for damping vibrations of bodies, U.S. Patent No. 989958, p. 3576-3580, 1911.

[16] J. Ormondroyd, J.P. Den Hartog, The theory of the dynamic vibration absorber, Journal of Applied Mechanics, ASME, 50(7):9-22, 1928.

[17] J.E. Brock, A note on the damped vibration absorber, Journal of Applied Mechanics, ASME, 13(4):A-284, 1946.

[18] J.P. Den Hartog, Mechanical Vibrations, McGraw-Hill, 4th ed., 1956.

[19] T. Ioi, K. Ikeda, On the dynamic vibration damped absorber of the vibration system, Bulletin of the Japanese Society of Mechanical Engineering, 21(151):64-71, 1978.

[20] S.E. Randall, D.M. Halsted, D.L. Taylor, Optimum vibration absorbers for linear damped systems, Journal of Mechanical Design, ASME, 103(4):908-913, 1981.

[21] R. Rana, T.T. Soong, Parametric study and simplified design of tuned mass dampers, Engineering Structures, 20(3):193-204, 1998.

[22] S.V. Bakre, R.S. Jangid, Optimum parameters of tuned mass damper for damped main system, Structural Control and Health Monitoring, 14(3):448-470, 2006.

[23] S. Krenk, J. Høgsberg, Tuned mass absorbers on damped structures under random load, Probabilistic Engineering Mechanics, 23(4):408-415, 2008.

[24] G.B. Warburton, Optimum absorber parameters for various combinations of response and excitation parameters, Earthquake Engineering and Structural Dynamics, 10(3):381-401, 1982.

[25] T. Asami, O. Nishihara, A.M. Baz, Analytical solutions to $\mathrm{H}_{\infty}$ and $\mathrm{H}_{2}$ optimization of dynamic vibration absorber attached to damped linear systems, Journal of Vibrations and Acoustics, ASME, 124(2):284-295, 2002.

[26] A.Y.T. Leung, H. Zhang, Particle Swarm Optimization of tuned mass dampers, Engineering Structures, 31(3):715-728, 2009.

[27] G.W. Housner et al., Structural Control: Past, Present and Future, Journal of Engineering Mechanics, 123(2):897-971, 1997.

[28] T.T. Soong, B.F. Spencer, Supplemental energy dissipation: state-of-the-art and state-ofthe-practice, Engineering Structures, 24(3):243-259, 2002.

[29] L. Kela, P. Vahaoja, Recent Studies of Adaptive Tuned Vibration Absorbers/Neutralizers, Applied Mechanics Reviews, 62(6), 9 pages, 2009.

[30] T. Pinkaew, Y. Fujino, Effectiveness of semi-active tuned mass dampers under harmonic excitation, Engineering Structures, 23(7):850-856, 2001.

[31] A.M. Aly, Proposed robust tuned mass damper for response mitigation in buildings exposed to multidirectional wind, The Structural Design of Tall and Special Buildings, 23(9):664691, 2014.

[32] C.-H. Loh, C.-H. Chao, Effectiveness of active tuned mass damper and seismic isolation on vibration control of multi-storey building, Journal of Sound and Vibration, 193(4):773-792, 1996.

[33] S.K. Rasouli, M. Yahyai, Control of response of structures with passive and active tuned mass dampers, The Structural Design of Tall Buildings, 11(1):1-14, 2002.

[34] P. Bisegna, G. Caruso, Closed-form formulas for the optimal pole-based design of tuned mass dampers, Journal of Sound and Vibration, 331(10):2291-2314, 2012.

[35] M. Abé, T. Igusa, Semi-active dynamic vibration absorbers for controlling transient response, Journal of Sound and Vibration, 198(5):547-569, 1996.

[36] A.G. Piersol, T.L. Paez, Harris' Shock and Vibration Handbook, McGraw-Hill, 6th ed., 2010.

[37] S.S. Rao, Mechanical Vibrations, Pearson, 5th ed., 2011.

[38] R. Villaverde, L.A. Koyama, Damped resonant appendages to increase inherent damping in buildings, Earthquake Engineering and Structural Dynamics, 22(6):491-507, 1993. 
[39] Sadek F, Mohraz B, Taylor AW, Chung RM. "A method of estimating the parameters of Tuned Mass Dampers for seismic applications", Earthquake Engineering and Structural Dynamics, 26(6):617-635, 1997.

[40] K. Ogata, Modern Control Engineering, Prentice Hall, 5th ed., 2009.

[41] C.R. Fuller, S.J. Elliott, P.A. Nelson, Active Control of Vibration, Academic Press, 1st ed., 1996.

[42] T.T. Soong, Active structural control in civil engineering, Engineering Structures, 10(2):7484, 1988.

[43] K.C.S. Kwok, B. Samali, Performance of Tuned Mass Dampers under wind loads, Engineering Structures, 17(9):655-667, 1995.

[44] M. Al-Dawod, B. Samali, J. Li, Experimetal verification of an active mass driver system on a five-storey model using a fuzzy controller, Structural Control and Health Monitoring, 13(5):917-943, 2006.

[45] F. Kerber, S. Hurlebaus, B.M. Beadle, U. Stöbener, Control concepts for an active vibration isolation system, Mechanical Systems and Signal Processing, 21(8):3042-3059, 2007.

[46] D.S. Nyawako, P. Reynolds, Response-dependent velocity feedback control for mitigation of human-induced floor vibrations, Smart Materials and Structures, 18(7):1-14, 2009.

[47] The MathWorks Inc., MATLAB User's Guide and Optimization Toolbox, USA, 2011. 